\title{
Freezing of a Lennard-Jones Fluid: From Nucleation to Spinodal Regime
}

\author{
Federica Trudu, Davide Donadio, and Michele Parrinello \\ ETH Zurich, Department of Chemistry and Applied Biosciences, clo USI-Campus, \\ via Giuseppe Buffi 13, CH-6900 Lugano, Switzerland \\ (Received 20 March 2006; published 6 September 2006)
}

\begin{abstract}
Using molecular dynamics, we investigate the crystal nucleation in a Lennard-Jones fluid as a function of the degree of supercooling. At moderate supercooling, a nucleation picture applies, while for deeper quenches, the phenomenon progressively acquires a spinodal character. We show that in the nucleation regime, the freezing is a two-step process. The formation of the critical nucleus is indeed preceded by the abrupt formation of a precritical crystallite from a density fluctuation in the fluid. In contrast, as the degree of supercooling is increased, crystallization proceeds in a more continuous and collective fashion and becomes more spatially diffuse, indicating that the liquid is unstable and crystallizes by a spinodal mechanism.
\end{abstract}

DOI: 10.1103/PhysRevLett.97.105701

In the absence of nucleation centers, moderately supercooled liquids are metastable, but after a finite time undergo homogeneous nucleation. This is usually described in terms of classical nucleation theory (CNT) in which spontaneous fluctuations lead to the formation of small crystallites $[1,2]$. When a crystallite exceeds a critical size, i.e. when it overcomes the nucleation free energy barrier, the whole system crystallizes. In this picture, the critical nucleus is assumed spherical, and the nucleation barrier, which depends only on its size, is determined by a balance between surface and volume free energy terms. Several computer simulations have helped to elucidate the microscopic aspects of crystal nucleation for moderate supercooling and allowed free energy barriers and nucleation rates to be computed for model systems such as hardspheres [3,4] and Lennard-Jones (LJ) [5-8]. These models are representative of real systems, e.g. colloids or globular proteins, and the theoretical predictions can be verified experimentally $[9,10]$. Nevertheless, calculations, as well as experiments, have proven challenging, and a comprehensive picture of crystallization kinetics and thermodynamics as a function of the degree of supercooling is still lacking. At deep quenches, the nucleation process was predicted to be affected by the proximity of a "pseudospinodal" [11,12], and earlier simulations of the freezing of LJ fluid supported this prediction [7,13], although the existence of a spinodal singularity for crystallization has not been proven yet.

In this Letter, we present a molecular dynamics (MD) study of the crystallization of a LJ fluid as a function of the degree of supercooling. The aim of this study is to ascertain to what extent CNT applies and to investigate how the degree of supercooling affects the crystallization process. We simulate an Argon fluid as described by an LJ potential [14] at temperature $T / T_{\text {melt }}=0.8$ and pressure $P=$ 0.25 kbar. We study a system of 6912 particles with periodic boundary condition (PBC). This system size is much larger than the critical nucleus, hence PBC will not affect its formation. Temperature and pressure are controlled by a
PACS numbers: 64.60.Qb, 07.05.Tp, 82.20.-w

thermostat [15] and a barostat [16]. We checked that during crystallization, pressure and temperature do not exhibit anomalous fluctuations. At low supercooling, crystallization takes place in a time scale much longer than present day MD time scale. Much technical progress has recently been made in this field, and using methods such as transition path sampling (TPS) [17], it is now possible to generate real dynamical trajectories in a computationally affordable time. In practice, we start from a crystallization trajectory, and at selected points along the path, the velocities are changed randomly while keeping the total kinetic energy constant. The maximum allowed change in velocity is $5 \%$. By propagating forward and backward in time from these altered points, new trajectories are generated. The ones that lead to crystallization are accepted and are used as new starting points. By iterating this procedure, we were able to harness ten statistically independent crystallization trajectories.

Along these trajectories, we perform a commitment probability analysis (CPA) [17], namely, we measure the probability that the system returns to the liquid state or proceeds toward crystallization when the particle velocities are changed and randomly chosen from the appropriate Maxwell distribution. The transition state ensemble consists of the configurations, in which nuclei are equally likely to promote crystallization or to dissolve. In transition state theory, this condition coincides with the maximum of the free energy along the proper reaction coordinate. We define a particle as solid-like, computing the dot product between its local $\mathbf{q}_{6}$ vector, i.e. the expansion in spherical harmonics of the nearest neighbors bonding vectors (see Ref. [7] for details), with the ones of the neighboring atoms, normalized on the number of neighbors. We have verified that the distributions of this quantity in the liquid and in the solid are neatly separated. A broad distribution of the size of critical nuclei is found: $n^{*}=240 \pm 34$ atoms, which is consistent with earlier studies [8]. We can now examine whether CNT is able to describe this phenomenon. In CNT, the free energy of formation of a 
crystalline nucleus is given by

$$
\Delta G=n \Delta \mu+\gamma S,
$$

where $\Delta \mu$ is the difference of the chemical potentials in the solid and in the liquid phase, and $\gamma$ is the interface free energy, while $S$ is the nucleus surface area. Assuming, as it is done in CNT, that the nucleus is spherical and has the density of the fcc crystal $\left(\rho_{\mathrm{fcc}}\right)$, then $S=(4 \pi)^{1 / 3} \times$ $\left(3 n / \rho_{\mathrm{fcc}}\right)^{2 / 3}$, and $\Delta G$ is a function of $n$ only. The CNT curve of $\Delta G(n)$, obtained using for $\Delta \mu$ and $\gamma$ the values computed for the same LJ system reported in the literature [7,18], predicts $n^{*} \sim 80$ and a corresponding critical value for the free energy $\Delta G^{*}=18.5 k_{B} T$ (Fig. 1). For the LJ system, as well as for spherical colloids, CNT is known to largely underestimate the size of the critical nuclei and their work of formation by about $40 \%[3,4,8]$, and it is not a surprise that our dynamically estimated $n^{*}$ is so much larger than 80 . We believe that a major cause for the failure of CNT is the assumption of spherical nucleus. In fact we find, in agreement with Ref. [8], that the crystallites are far from spherical, especially the smaller ones, and Eq. (1) needs to be corrected for this effect. In order to take this effect into account, we assume that the crystallites have an ellipsoidal form with axes determined from the inertia tensor [19]. A measure of the anisotropy is given by the ratio between the longest and shortest inertia tensor axis that fluctuates around $3 / 2$. We use this ellipsoidal approximation to evaluate directly the surface of the crystallites observed in the MD simulation, and their work of formation is computed using this estimate of $S$ in Eq. (1) (Fig. 1). The values provided by our "extended CNT" model,

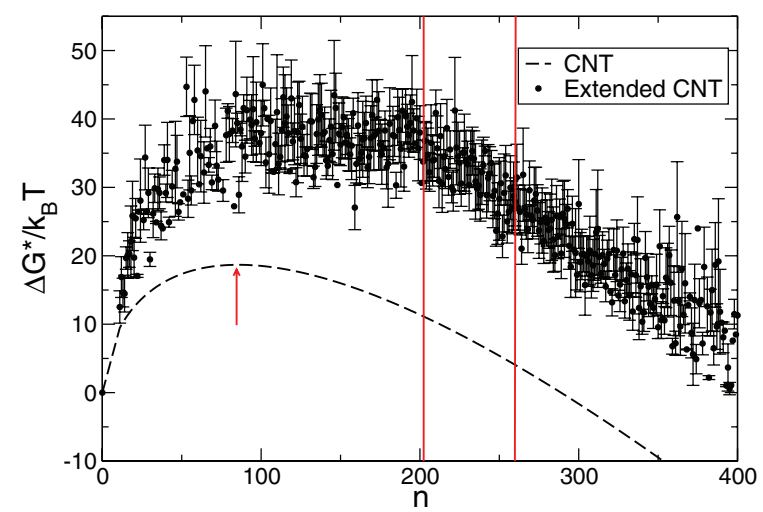

FIG. 1 (color online). Comparison between the work of formation $\Delta G$ of nuclei of size $n$ as predicted by CNT (dashed line) and using in Eq. (1) the values of $S$ computed by the ellipsoidal approximation along the reactive trajectories (dots with error bars). For a given $n$, different values of $S$ are possible. The corresponding values of $\Delta G$ computed from Eq. (1) are averaged and their root mean square deviations reported in the picture. The values $\Delta \mu=0.262 \mathrm{~kJ} / \mathrm{mol}$ [7] and $\gamma=\gamma(111)=$ $0.0307 \mathrm{~kJ} / \mathrm{mol} / \AA^{2}$ [18] have been used. The transition state ensemble, identified by the CPA on the MD trajectory, is delimited by the vertical lines. although rather scattered, give a much better estimation of $n^{*}$ and $\Delta G^{*}$. They show that the effect of considering a more realistic approximation of the shape of the crystalline nuclei almost entirely accounts for the discrepancy between CNT and simulations, while, at the same time, they suggest that close to coexistence, the main features of nucleation are captured by classical thermodynamic models.

The cost of creating an interface between solid and liquid plays a central role in nucleation. It is therefore interesting to investigate how this quantity varies during the nucleation process. Surface free energies are difficult to evaluate, but one can monitor related quantities such as the average coordination of the solid-like particles with other solid-like particles $\left(c_{s s}\right)$ [20]. We analyze the time evolution of this parameter, which directly takes into account the size of the solid-like particles and indirectly the nature of the crystalline-liquid interface. On average in the liquid phase, a small fraction of particles $(0.5 \%)$ with a solid-like environment are found randomly in the simulation box. In this regime, the value of $c_{s s}$ is close to zero, until nucleation is initiated and a cluster of about 60 solid-like particles with an average local specific volume $\left(\sim 40 \AA^{3} /\right.$ atom $)$ close to the crystalline phase is formed as a consequence of a local statistical fluctuation. At $T / T_{\text {melt }}=0.8$, the fingerprint of this event is a jump in $c_{s s}$ (Fig. 2), which indicates that the formation of a crystalline embryo, able to grow to the critical size, is not a gradual process. This is a dynamical bottleneck that the system has to overcome in order to initiate the crystallization. This event could not have been detected by monitoring the number of solid-like particles in the liquid, which grows more slowly and is a much less sensitive indicator. These precursors are still much smaller than the critical nuclei and indeed still have a low probability $(<0.2)$ to evolve toward crystallization. This novel result suggests that at moderate supercooling, crystallization is a two-step process characterized by the rapid formation of under-critical precursors fol-

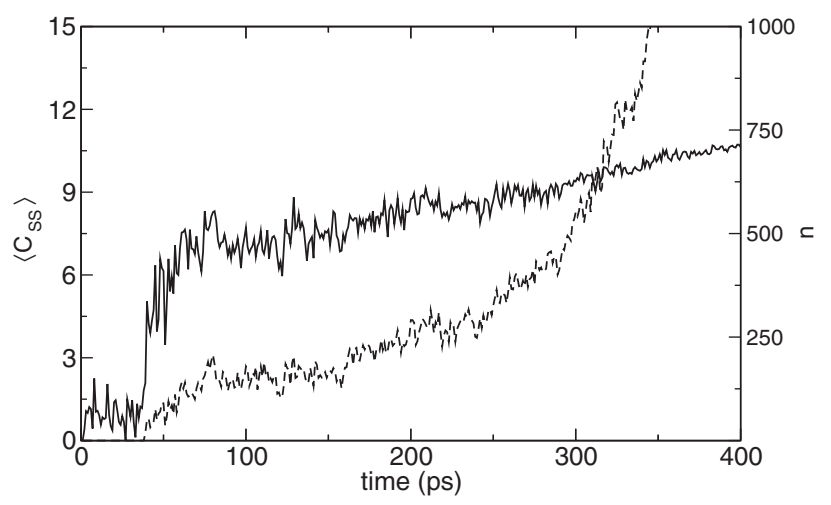

FIG. 2. The number of solid-like particles in the largest cluster (dashed line) and the average coordination between solid-like particles $\left(c_{s s}\right.$, solid line $)$ at $T / T_{\text {melt }}=0.8$. 
lowed by a slower growth that, if the critical size is reached, will lead to crystallization.

We now turn to the study of nucleation as a function of the degree of supercooling. In order to do this, a computationally more efficient method than TPS is needed, especially to evaluate $\Delta G^{*}$. We use the metadynamics (MTD) method [21,22], which is based on a coarse-grained dynamics in the space of few reaction coordinates, biased by a history-dependent potential which drives the system toward the lowest transition state, thus allowing for an efficient exploration of the free energy surface. This method relies on the identification of the appropriate reaction coordinates, which are obtained and validated from the unbiased trajectories generated by TPS. Previous experience has shown that collective coordinates that describe the global crystalline order are not appropriate [23]. For this reason, we define an indicator of local crystalline order by arbitrarily selecting a particle and measuring the Steinhardt order parameter $Q_{6}$ [24] of a subset of the $N_{\text {sub }}$ nearest neighbors of the tagged particle. In this calculation, we take $N_{\text {sub }}=350$ [25]. The local $Q_{6}$ is coupled with the potential energy of the system as a second reaction coordinate. In these simulations, the history-dependent potential is made of Gaussians, whose height and width have been tuned so to achieve an accuracy of $\sim 2 k_{B} T$ in the computation of the free energy barrier [22]. The historydependent potential is made of Gaussians $1.5 k_{B} T$ high put every $4 \mathrm{ps}$. The Gaussian width is 0.01 for $Q_{6}$ and $40 \mathrm{~kJ} / \mathrm{mol}$ for the potential energy. In these simulations, the temperature and the pressure of 0.25 kbar are enforced by the Berendsen thermostat and barostat [26].

In the range of temperatures between 0.7 and $0.8 T / T_{\text {melt }}$, the crystallization process evolves first along the local order parameter and only later a variation in the potential energy of the system is observed. At $T / T_{\text {melt }}=0.8$, MTD yields a free energy barrier $\Delta G^{*}=35.4 \pm 2.0 k_{B} T$, which agrees with the evaluation obtained by the application of the empirical model to the TPS trajectories. The temperature dependence of $\Delta \mu$ as in Ref. [7] has been used, while $\gamma$ is assumed constant for such small variations of $T$ [18]. A further confirmation of the validity of these reaction coordinates can be obtained by repeating the MTD at $T / T_{\text {melt }}=0.75$. The value $\Delta G^{*}=23.3 \pm 2.0 k_{B} T$ thus obtained is remarkably consistent with the one computed in Ref. [8] at the same supercooling, but at different pressure. This provides a strong cross validation of both the extended CNT model and the choice of reaction coordinates in the MTD runs. In order to locate the transition state, the CPA of the MTD trajectories is performed. We calculated $\Delta G^{*}$ of these transition state ensembles using our extended CNT model. The results in Fig. 3 show an excellent agreement between MTD and phenomenological model at moderate supercooling, down to $T / T_{\text {melt }}=0.75$. Below this temperature, large fluctuations are observed in the number of solid-like particles at the transition state and consequently in the activation barrier as estimated by our empirical

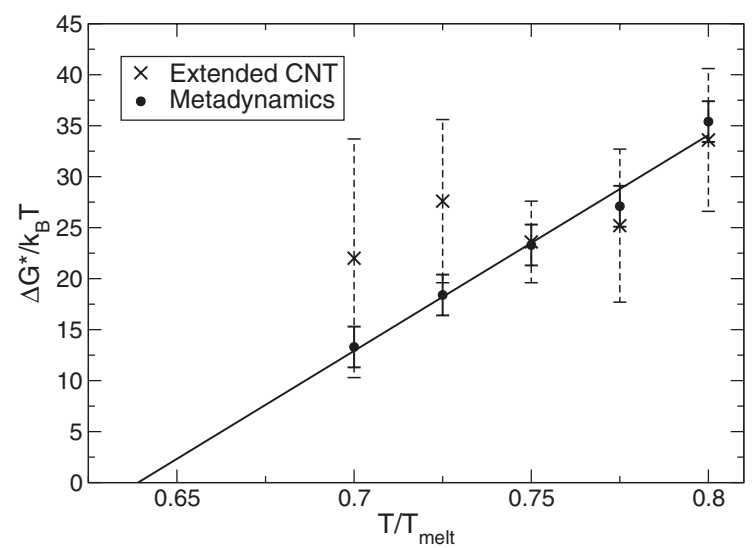

FIG. 3. The free energy barriers as computed from MTD runs at different temperatures (circles) are compared to the work of formation of the critical nuclei and as predicted within our extended CNT model (crosses with dashed error bars). A linear fit to the MTD data is also shown.

model. At higher supercooling, a breakdown of the model occurs, signaling that freezing proceeds via a process different from nucleation. This is to be expected since mean-field theories $[11,27,28]$ predict that the nucleation barrier vanishes at large supercooling where a pseudospinodal singularity influences nucleation. They also predict that the interface of the critical nucleus becomes very broad and eventually diverges at the spinodal [27,28]. Pioneering simulations have suggested that at high supercooling, crystallization may occur by a collective mechanism [13], but no definitive proof of the existence of a spinodal line for LJ crystallization has been given yet.

A linear extrapolation of the MTD data predicts that $\Delta G^{*}$ vanishes at $T / T_{\text {melt }}=0.64$ (Fig. 3). To verify that in this supercooling range a spinodal instability sets in, we have extended our study of nucleation to deep quenches, up to $T / T_{\text {melt }}=0.6$. At first, we have performed two sets of standard MD simulations at $T / T_{\text {melt }}=0.65$ and 0.7 . In these runs, crystallization is achieved spontaneously. The average time lag between quench and crystallization are $0.17 \pm 0.05 \mathrm{~ns}$ and $2.3 \pm 0.8 \mathrm{~ns}$ at $T / T_{\text {melt }}=0.65$ and 0.7 , respectively. This suggests indeed that $\Delta G^{*}$ is very low. The progression from standard nucleation to spinodal instability is illustrated in Fig. 4. The nucleation center is ellipsoidal and compact at $T / T_{\text {melt }}=0.8$. It has a less compact character and it is accompanied by smaller nuclei in the intermediate range $T / T_{\text {melt }}=0.7$. Finally at $T / T_{\text {melt }}=0.65$, it has a branched fractal character with a very broad specific surface. It is worth noting that in all these MD runs, the system never amorphizes, as the single component LJ fluid is not a glass former. In MTD runs at deep quenches $\left(T / T_{\text {melt }}=0.65\right.$ and 0.6$)$, where the abovementioned set of order parameters is adopted, the basin corresponding to the liquid state is absent, and the transformation involves a variation of the potential energy of the 


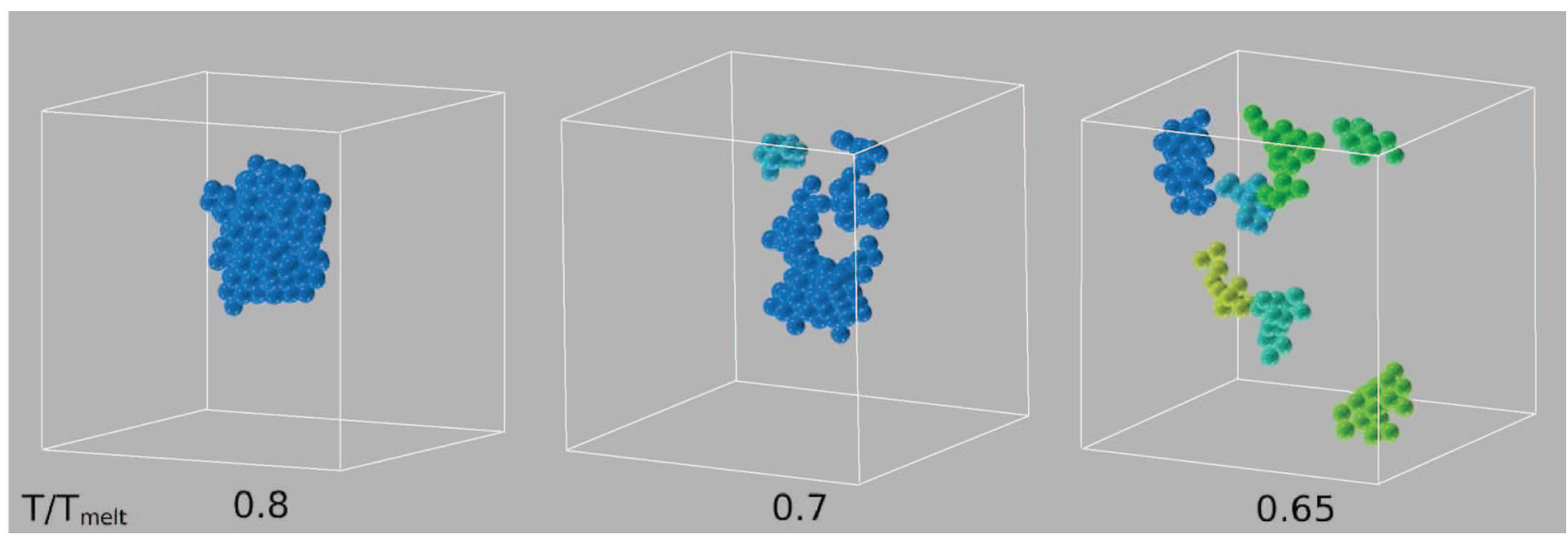

FIG. 4 (color). For $T / T_{\text {melt }}=0.8$, a snapshot of a critical nucleus is shown. For $T / T_{\text {melt }}=0.7$ and 0.65 , we have chosen a representative frame along the spontaneous crystallization trajectories. Colored spheres represent particles with a solid-like environment. Different colors indicate different aggregates with solid-like character. For clarity, the liquid-like particles are not shown.

system since the beginning. This evidence suggests that the liquid becomes unstable, rather than metastable with respect to the solid, and crystallization turns into a collective process.

In summary, we have presented an analysis of the temperature-dependent behavior of crystallization in a $\mathrm{LJ}$ fluid. Our results demonstrate that for shallow supercooling, CNT captures the essential thermodynamic features of freezing, i.e. the height of the nucleation barrier and the size range of the critical nucleus, provided that the approximation of spherical nucleus is abandoned. Nonetheless, the kinetics of the initial stage of the growth of crystalline embryos is characterized by the sudden formation of dense crystalline cluster of finite size. This feature was not predicted by classical kinetic theories that describe nucleation in terms of addition and removal of single particles [29]. It is however consistent with nucleation seen near spinodals in Ising models with long-range interactions [30]. At lower temperatures, we observed a crossover from a classical nucleation regime to a more collective mechanism of freezing, influenced by the existence of a spinodal singularity. The presence of a spinodal effect in the crystallization from the liquid, as predicted by a field theory approach [11], is here confirmed.

We thank R. Martonak, A. Laio, Y. Mantz, D. Moroni, and V. Buch for useful discussions, and M. Valle for the development of helpful visualization tools.

[1] D. W. Oxtoby, Adv. Chem. Phys. 70, 263 (1988).

[2] K. F. Kelton, Solid State Phys. 45, 75 (1991).

[3] S. Auer and D. Frenkel, Nature (London) 409, 1020 (2001).

[4] S. Auer and D. Frenkel, Nature (London) 413, 711 (2001).

[5] P. R. ten Wolde and D. Frenkel, Science 277, 1975 (1997).

[6] W. C. Swope and H.C. Andersen, Phys. Rev. B 41, 7042 (1990).
[7] P. R. ten Wolde et al., J. Chem. Phys. 104, 9932 (1996).

[8] D. Moroni et al., Phys. Rev. Lett. 94, 235703 (2005).

[9] P. Wette et al., J. Chem. Phys. 123, 174902 (2005).

[10] U. Gasser et al., Science 292, 258 (2001).

[11] W. Klein and F. Leyvraz, Phys. Rev. Lett. 57, 2845 (1986).

[12] W. Klein et al., Phys. Rev. Lett. 85, 1270 (2000).

[13] J. X. Yang et al., Phys. Rev. Lett. 60, 2665 (1988).

[14] The interaction potential cutoff is set at $9 \AA$ and longrange corrections are considered in the computation of both total energy and pressure.

[15] W. G. Hoover, Phys. Rev. A 31, 1695 (1985).

[16] M. Parrinello and A. Rahman, Phys. Rev. Lett. 45, 1196 (1980).

[17] P. Bolhuis et al., Faraday Discuss. 110, 421 (1998).

[18] J. R. Morris and X. Song, J. Chem. Phys. 119, 3920 (2003).

[19] The eigenvalues $I_{j}$ of the inertia tensor of a scalene ellipsoid of mass $m$ are related to its semiaxes $\left\{a_{i}\right\}$ by $I_{j}=$ $m\left(a_{i}^{2}+a_{k}^{2}\right) / 5$, where $i, k \neq j$.

[20] Two particles are considered nearest neighbors if their distance is smaller than $5.1 \AA$, corresponding to the first minimum of the radial distribution function of the liquid.

[21] A. Laio and M. Parrinello, Proc. Natl. Acad. Sci. U.S.A. 99, 12562 (2002).

[22] P. Raiteri et al., J. Phys. Chem. B 110, 3533 (2006).

[23] J.-M. Leyssale et al., J. Chem. Phys. 122, 104510 (2005).

[24] P. J. Steinhardt et al., Phys. Rev. B 28, 784 (1983).

[25] The arbitrary choice of a particle as nucleation center artificially restricts the number of possibilities through which nucleation can occur: so the estimate of $\Delta G^{*}$ has to be corrected by an entropic factor defined as $-k_{B} T \ln \left(N_{\text {sub }} / N\right)$, where $N$ is the total number of particles in the simulation box.

[26] H. J. C. Berendsen et al., J. Chem. Phys. 81, 3684 (1984).

[27] J. W. Cahn and J.E. Hilliard, J. Chem. Phys. 31, 688 (1959).

[28] C. Unger and W. Klein, Phys. Rev. B 29, 2698 (1984).

[29] R. Becker and W. Doering, Ann. Phys. (Leipzig) 24, 719 (1935).

[30] L. Monette and W. Klein, Phys. Rev. Lett. 68, 2336 (1992). 